\title{
Measuring distortion of skeletal elements in Lodox Scatscan-generated images
}

\author{
Kyra E. Stull, MS ${ }^{1}$, Ericka N. L'Abbé, PhD, D-ABFA ${ }^{1}$, and Stef Steiner, BSc ${ }^{2}$ \\ ${ }^{1}$ Department of Anatomy, University of Pretoria, Private Bag x323, Arcadia, 0084, South Africa \\ ${ }^{2}$ Lodox Systems (Pty) Ltd and MRC/UCT Medical Imaging Research Unit (MIRU), Department of Human Biology, \\ Faculty of Health Sciences, University of Cape Town, Observatory, 7925, South Africa
}

\section{Original Communication}

4 Figures; 2 Tables

Abbreviated title: Distortion in Lodox Statscan images

Corresponding Author:

Kyra E. Stull, MS

Department of Anatomy

University of Pretoria

Private Bag x323, Arcadia, 0084

Email: kstullster@gmail.com

Telephone: +270123192438

Cell Phone (South Africa): +27 799840465

Fax South Africa: +270123192240

\begin{abstract}
Due to a scarcity of available skeletal material, anthropologists and other practitioners face difficulties with either the creation or validation of techniques used to estimate a biological profile in subadults. In order to address this problem, radiographic images of living individuals are often used in lieu of dry skeletal elements. However, radiographic images suffer from distortion. Some problems with metric
\end{abstract}


analyses of radiographic images may be addressed with the Lodox Statscan, an X-ray machine that claims to produce minimal distortion along the scan-axis due to a linear slot-scanning design. The purpose of this research was to measure the distortion of skeletal elements in radiographic images generated from a Lodox Statscan. Skeletal elements subject to multiple imaging variables that affect distortion were radiographed, measured, and then compared to the dry bone measurements through multiple approaches. An $85 \%$ percent agreement was obtained within a $+/-1 \mathrm{~mm}$ range and a $97 \%$ agreement within a $+/-2 \mathrm{~mm}$ range. Percent difference results demonstrate that slot-axis measurements incurred more distortion than scan-axis measurements (11.8\% and 2.7\%, respectively). Inclusion of foam results in $4.5 \%$ more error than when foam is not included in the image. Angled scan-axis measurements also incurred more distortion than either non-angled slot- and scan-axis measurements. A Bland-Altman plot reveals an overall agreement between the radiographic and dry bone measurements, with most measurements falling within the upper and lower limits. Similar measurement error is found in Statscan radiographic and dry bone measurements; therefore, the Statscan offers a radiographic venue to collect metric data.

Key Words: radiographs; precision; percent difference

\section{INTRODUCTION}

The absence of subadult remains in current skeletal collections is a major research obstacle for anthropologists and other practitioners in human biology and anatomy. Current collections contain temporally older subadult skeletons which are inappropriate for developing models for modern groups. Furthermore, the collections do not contain enough individuals per age category for sound statistical analyses; this is especially true when ancestry and sex are used to further separate groups. Under these circumstances, a sample may be reduced to one or two individuals per age and ancestry category and, as expected, cannot be used to represent the normal range of human variation. 
Radiographic images can be used either in lieu of, or in conjunction with, dry skeletal material. Previous research which utilized radiographic images generally focused on observational analyses (Hill, 1939; Greulich and Pyle, 1959; Lewis and Garn, 1960; Buikstra and Ubelaker, 1994; Ontell et al., 1996; Scheuer and Black, 2000; Crowder and Austin, 2005; AlQahtani et al., 2010). Studies which collected metric data from radiographic images noted distortion rates between 1 and 3\% for skeletal elements with minimal object-image distance (OID) and great source-image distance (SID) in images generated within a prospective longitudinal study (Maresh, 1955, 1970; Anderson et al., 1964; Gindhart, 1973; Buschang, 1982; Smith and Buschang, 2004; Smith, 2007). Prospective research designs using radiographic images eliminate the issue of unknown SID and OID and/or create operating procedures in order to produce the least distortion; however, active prospective longitudinal radiographic studies rarely exist. In retrospective radiographic studies, the SID and OID are unlikely to be known. Therefore, radiographs, which are taken daily in hospitals and forensic laboratories, may be an impracticable source for metric variables as the magnitude of the distortion is not considered in each image.

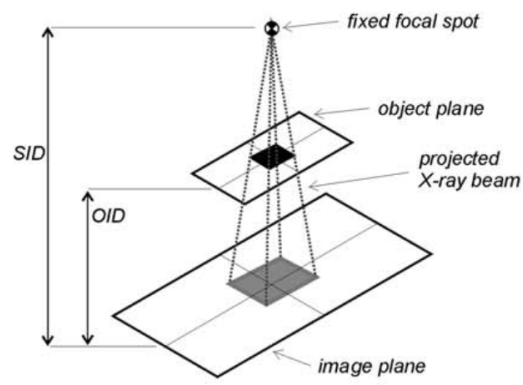

a) original configuration OID and SID

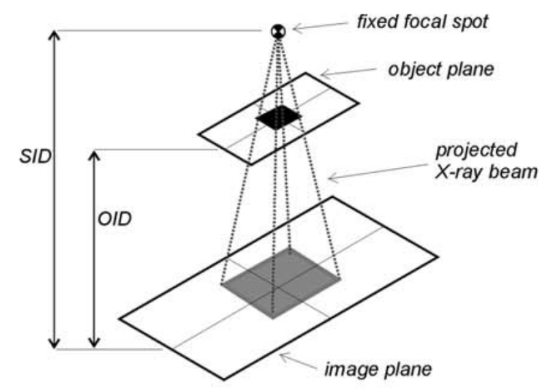

b) increasing OID

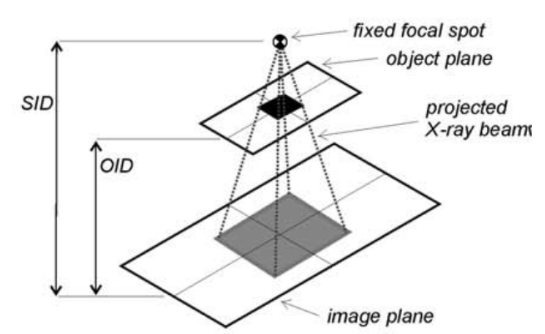

c) decreasing SID

Figure 1 - Illustration of the relationship between image distortion for a) a set SID and OID, b) an increased OID, and c) a decreased SID.

Distortion is defined as "the misrepresentation of object size or shape as projected onto the radiographic recording medium" (Bontrager, 2001). Distortion and magnification in conventional radiographs is inherent and is greatly dependent on both the SID and the OID (Figure 1) (Bontrager, 2001). 
A greater SID results in less distortion (Bontrager, 2001). When generating a radiographic image using a cone-beam, the final image contains distortion diverging from the center, such that objects closer to the center of the beam (central ray) are less distorted than objects further from the center (Bontrager, 2001; Carleton and Adler, 2001). The shorter the SID, the greater the divergence angle of the X-ray beams and the greater the distortion; conversely, a greater SID allows for less divergence of the beams, thus less distortion (Figure 1). Most conventional radiographic machines use a cone beam. In contrast, distortion from a collimated fan beam occurs only in the slot (perpendicular) direction of the beam, not in the scan (parallel) direction (see below). A second factor that produces distortion in radiographic images is OID (Figure 1). The closer the object is to the image plane, the less its projected image is distorted because peripheral X-rays diverge less from the central ray of the X-ray beam before they reach the image plane. Image receptors immediately adjacent to the object increase image sharpness and decrease distortion. Therefore, a combination of a smaller OID and a greater SID results in the least distortion (Bontrager, 2001; Carleton and Adler, 2001).

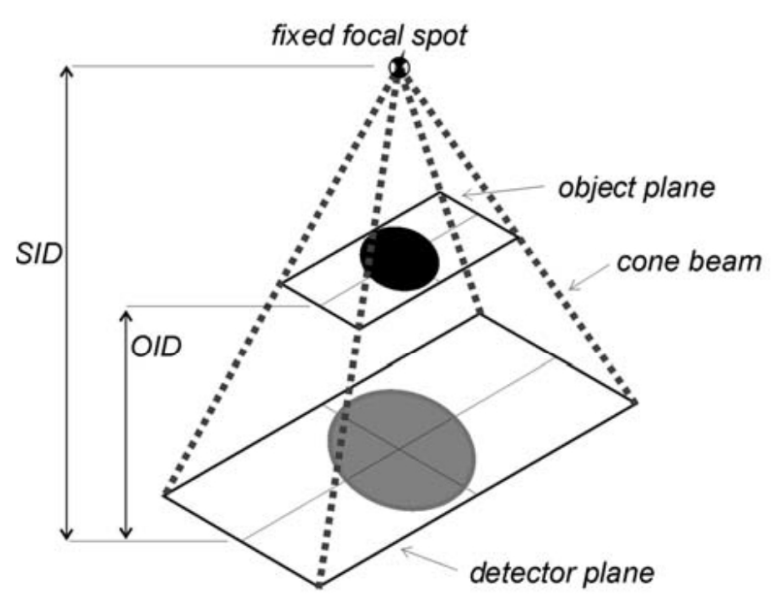

a) Full-field

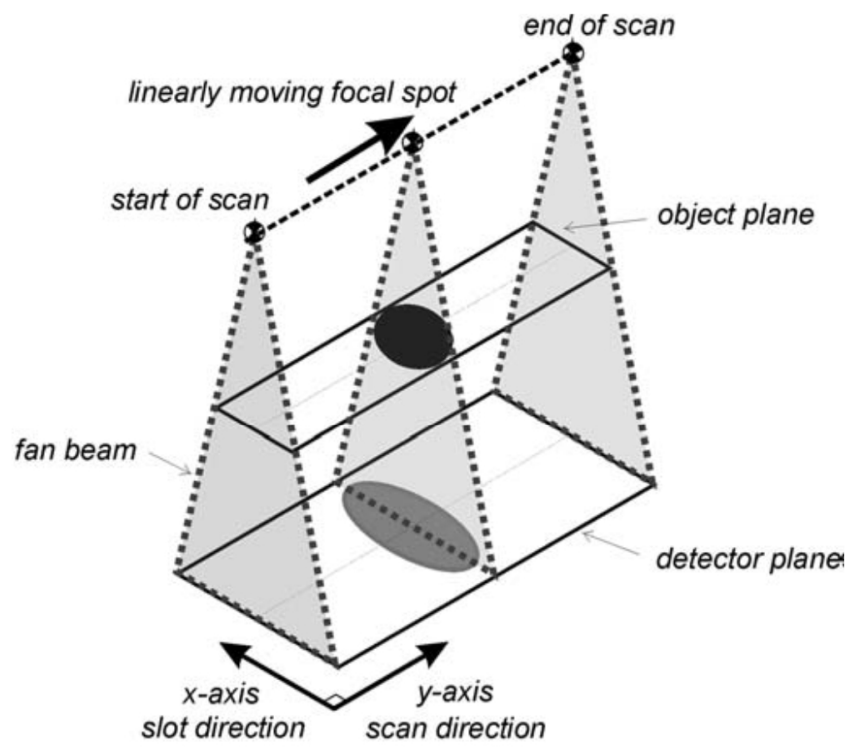

b) Slot-scanning

Figure 2 - Illustration of differences in distortion due to cone beam (a) and fan-beam (b) geometry. 
As can be seen in Figure 2a, the cone beam of a full-field X-ray system produces distortion in both the $\mathrm{x}$ - and the $\mathrm{y}$-direction of the object on the image plane. The distortion is related to the SID and OID. Simple triangle geometry can be used to determine the extent of the distortion, provided both SID and OID are known. However, SID and OID are rarely recorded and not likely to be known when retrospectively evaluating conventional radiographs. Use of the Lodox Statscan radiographic images may eliminate the problems associated with metric data collection because of the claim to generate minimal distortion in the $y-$ axis (scan direction). The machine is designed to offer rapid image acquisition (10-13 seconds), to produce a highly detailed whole-body image, and to emit extremely low radiation dosage to patients and

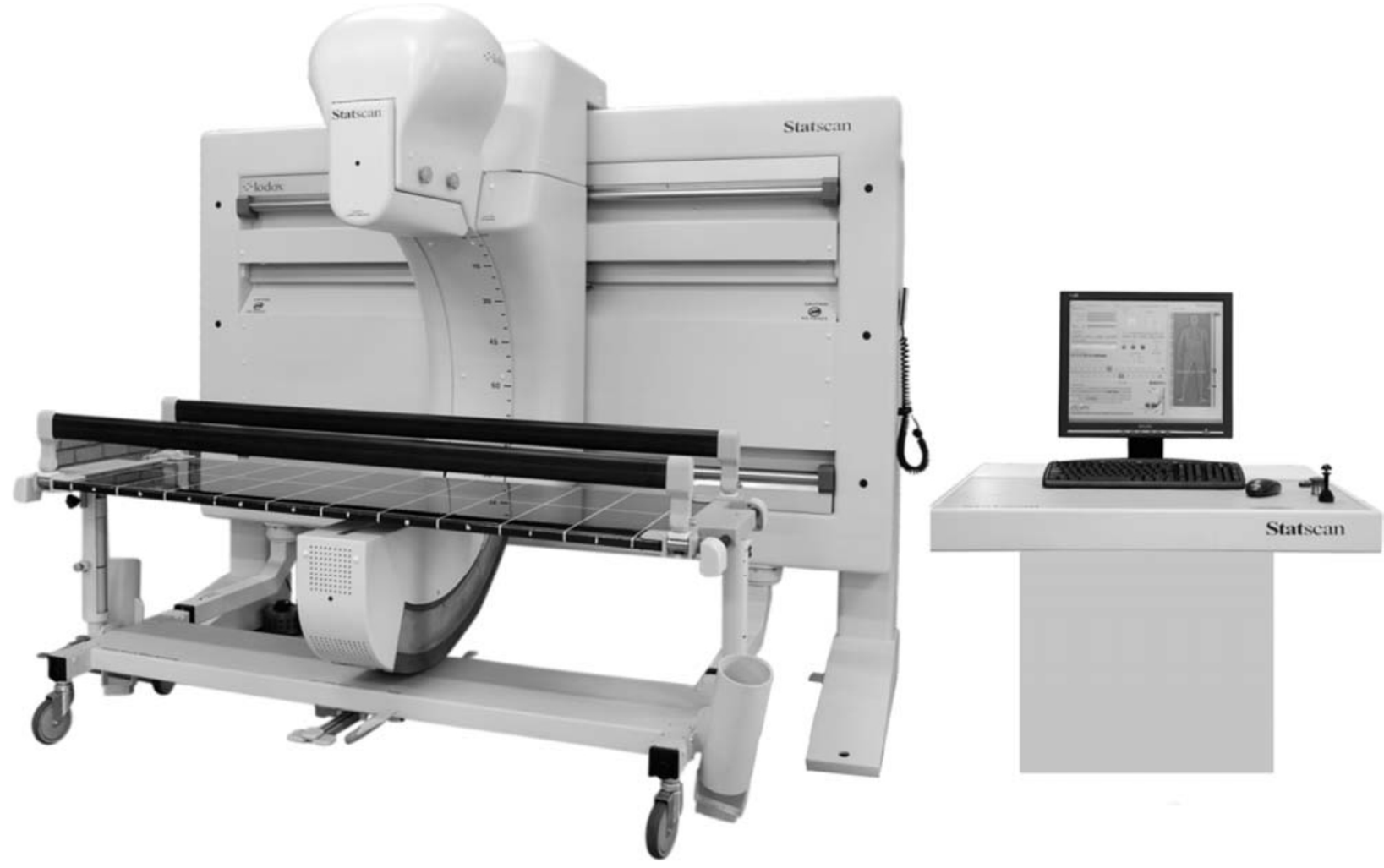

Figure 3 - An image of a Lodox Statscan X-ray machine.

operators, thus making the machine safer to use than conventional radiography equipment (Knobel et al., 2006; Evangelopoulos et al., 2009). The Statscan has an X-ray tube mounted on one end of a C-arm and an image receptor on the other end (Figure 3 ). In order to generate a radiographic image, the C-arm is linearly and continuously moved over an object along the y-axis with the beam perpendicular to the direction of 
motion. Furthermore, the $\mathrm{C}$-arm can rotate around the patient allowing for numerous views such as oblique, horizontal, and shoot-through (Beningfield et al., 2003). Unlike conventional full-field cone beam X-ray machines, the Statscan incorporates a tightly-collimated fan beam for scanning images. The fan beam is adjustable to the width of the table in the slot direction (approximately $680 \mathrm{~mm}$ wide), and is fixed in width in the scan direction (approximately $2 \mathrm{~mm}$ ).

Figure $2 \mathrm{~b}$ shows the Lodox Statscan fan-beam geometry. The X-ray source is a fixed distance from the image plane and moves linearly from the start to the end of the scan. Distortion of the object still occurs in the slot direction (x-axis), similar to full-field X-ray systems, but the narrow fan beam in the scan direction (y-axis) produces negligible distortion. Therefore, measurements taken along the scan direction of the image may be expected to represent the original dimensions of the object. The source-image distance for the Lodox machine is $130 \mathrm{~cm}$; the SID is greater than current standards and may aid in reducing distortion of the generated image along the x-axis. The top of the table, in the lowest position, is approximately $60 \mathrm{~mm}$ from the detector (imaging) plane and allows for a minimal OID. The Lodox Statscan is capable of producing images that are up to $1800 \mathrm{~mm} \times 680 \mathrm{~mm}$ in size. The fundamental pixel size is 60 microns and the contrast resolution is 14-bit greyscale, i.e., more than 16000 grey levels. The spatial resolution is as high as 5 linepairs $/ \mathrm{mm}$ but is dependent on the image settings.

Due to the design, the Lodox Statscan produces only x-axis distortion, and the images along the scan-axis (y-axis) may be accurately measured. The implications of images without distortion are enormous for anthropologists. To date, no published literature has examined distortion in skeletal elements of full body images using a Lodox Statscan. If validated, this will substantiate the application of osteometric techniques based on Lodox Statscan images to dry bone without a correction factor for distortion. The purpose of this study was to measure distortion in Lodox Scatscan-generated images of dry bone when subject to central or peripheral table placement, straight or angled table placement, and variable OID. 


\section{MATERIALS AND METHODS}

Femora and humeri from individuals aged less than 12 years were used. The skeletal elements are housed in the Human Skeletal Collection of the University of Cape Town, South Africa. The skeletal elements were in various developmental states with different overall sizes.

Four measurements were obtained from each humerus and femur using an osteometric board and sliding calipers. The humeral measurements include: maximum length (mxl), proximal breadth $(\mathrm{pb})$, distal breadth (db), and midshaft breadth (msb). The measurements were analogous for the femur with the exception of bicondylar length (bcl) which replaced proximal breadth. Three measurements (mxl, db, and msb) follow the standards of Fazekas and Kósa (1978) for immature remains. Two measurements (pb and bcl) are adopted from standards applied to mature remains (Moore-Jansen et al., 1994). Since the epiphyses were not fused, only diaphyseal dimensions were taken.

Six long bones were subjected to six rounds of imaging with a Lodox Statscan X-ray scanner (Lodox Systems, South Africa) located at the University of Cape Town in the Department of Human Biology, Faculty of Health Sciences. The SID was a fixed dimension of $130 \mathrm{~cm}$ and the table was in the lowest position, allowing a minimum OID of approximately $6 \mathrm{~cm}$. This is the recommended setting and is what is most commonly used. In order to account for soft tissue - a factor which affects OID (when the object is bone) - two different heights of dense foam were used, namely $25 \mathrm{~mm}$ and $50 \mathrm{~mm}$, to simulate different soft tissue thicknesses. The foam also increased the distance between the object and the image receptor (OID).

The long bones were subject to six imaging rounds, each with a different combination of imaging variables, namely 1) midline or peripheral table position, 2) angled or parallel position on table, and 3) with or without foam (Table 1). When the bone was angled, the most proximal portion was placed on the midline and the distal portion was pointed to the periphery. The angle of each bone was measured using the Lodox 
Table 1 - Imaging variables which each bone was subject per imaging round.

$\begin{array}{cccc}\begin{array}{c}\text { Imaging } \\ \text { Round }\end{array} & \begin{array}{c}\text { Midline (0) / } \\ \text { Peripheral (1) }\end{array} & \begin{array}{c}\text { Straight (0) / } \\ \text { Angled (1) }\end{array} & \begin{array}{c}\text { Foam (0) / } \\ \text { No Foam (1) }\end{array} \\ \mathbf{1} & 0 & 0 & 0 \\ \mathbf{2} & 1 & 0 & 0 \\ \mathbf{3} & 1 & 0 & 1 \\ \mathbf{4} & 1 & 0 & 1 \\ \mathbf{5} & - & 1 & 1 \\ \mathbf{6} & - & 1 & 1 \\ \text { '- if the bone was angled the proximal portion was midline and the distal portion } \\ \text { was towards the peripheral. }\end{array}$

imaging software (DVS 2.8.8.2, Lodox Systems, South Africa); the mean angle was $52^{\circ}$. The reasons for selecting various combinations of positions and foam dimensions was to test which combinations affected distortion and to correspond generated images of bones to actual patient positions from hospitals.

Long bone measurements were taken from the generated radiographic images using the Lodox imaging software following the same definitions as applied to the dry bone sample. This was possible as all measurement definitions require the dry bones to be in the anatomical position, which was how the bones were imaged and measured. Measurements of the Statscan-generated images were compared to the original dry bone measurements. Precision is the consistency of two measurements (Ousley, 1995; Ulijaszek and Kerr, 1999). Specifically for this study, measurement error due to distortion was examined; essentially, the precision of the measurements was tested. In order to assess the level of agreement between the measurements and ultimately the levels of distortion, different approaches were applied.

Percent agreement between the two measurement sets was first determined at the $+/-1 \mathrm{~mm}$ and then at the $+/-2 \mathrm{~mm}$ levels. The 1 and $2 \mathrm{~mm}$ error limits were chosen as these are considered acceptable levels of error within forensic anthropology. To obtain the percent agreement, the number of discordant pairs is subtracted from the number of in-agreement pairs and divided into the total number of pairs. Standards 
within forensic anthropology do not specify a particular agreement level to be accepted in the field; for this study a percent agreement over $75 \%$ is considered acceptable.

Due to size differences between the measurements (i.e., lengths versus breadths), calculation of the absolute differences of the measurements would be uninformative as a means to compare the results. For example, one millimeter difference in a long bone length of $150 \mathrm{~mm}$ has a different percentage of error than $1 \mathrm{~mm}$ difference in a midshaft measurement of $10 \mathrm{~mm}$. For these reasons, comparison of dry bone and radiographic measurements was evaluated through percent difference. Percent difference compares two calculated values to each another such that the absolute value of the differences of the measurements is computed and is divided into the mean of the two measurements (see below). Comparisons of percent differences were made between the $\mathrm{x}$ - and $\mathrm{y}$-axis measurements, central and peripheral placements, angled and straight placements, and the two foam sizes.

$$
\text { Percent difference }=\frac{x_{1}-x_{2}}{\left(\frac{x_{1}+x_{2}}{2}\right)} * 100
$$

A Bland-Altman plot depicts agreement between measurements. Although many studies evaluate measurement agreement of quantitative variables using a correlation coefficient, this approach reflects only the relationship of the two measurements, not the agreement between the two measurements (Bland and Altman, 1986). Measurements in low agreement may still produce high correlations. The Bland-Altman plot graphs the difference of the measurement against the mean which provides an illustration of the spread of differences in measurements. Essentially, the plot depicts precision of the two measurements. For the dry and radiographic measurements to be in sufficient agreement, we would expect the data to fall within the upper and lower standard deviations which can also be considered the upper and lower limits of agreement (Bland and Altman, 1986; Geeta et al., 2009). If the differences between the measurements generally fall within the two standard deviations, the Lodox Statscan images are considered interchangeable with dry bone for the purpose of measurement. 


\section{RESULTS}

Percent agreement with a $+/-1 \mathrm{~mm}$ range was fairly high at $85 \%$. The dry and radiographed bone measurements with a $+/-2 \mathrm{~mm}$ range resulted in an even higher agreement of $97 \%$. Percent differences were used to evaluate the error in the total sample, $x$ - and $y$-axis measurements, central and peripheral table placement, angled and straight table placement, and with or without foam. The total sample, inclusive of both $\mathrm{x}$ - and $\mathrm{y}$-axis measurements, ranged from 0.0 to $11.8 \%$ differences. $\mathrm{X}$-axis measurements demonstrated percent differences of $0.0-11.8 \%$, while y-axis measurements demonstrated percent differences of $0.0-$ 2.7\%. Centrally located $\mathrm{x}$-axis measurements had a percent difference range of 0.0 to $5.4 \%$ and peripherally located measurements ranged from 0.0 to $6.3 \%$. Only a $1 \%$ difference was found between central and peripheral placement in x-axis measurements. Centrally located y-axis measurements had a maximum percent difference of $0.56 \%$ and peripherally located y-axis measurements had a maximum percent difference of $0.44 \%$.

\section{Table 2 - Percent differences between $x$ - and $y$-axis measurements when imaged with and without foam} simulating OID.

$\begin{array}{ccc} & \begin{array}{c}\min \\ \text { \% difference }\end{array} & \begin{array}{c}\max \\ \text { \% difference }\end{array} \\ \mathrm{Y} \text { - no foam } & 0 & 1.2 \\ \mathrm{X} \text { - no foam } & 0 & 1.7 \\ \mathrm{Y} \text { - with foam } & 0 & 1.6 \\ \mathrm{X} \text { - with foam } & 1.7 & 6.25\end{array}$

The percent difference range for $\mathrm{x}$-axis measurements with foam was 1.7 to $6.25 \%$ while the $\mathrm{x}$-axis measurements without foam were 0.0 to $1.7 \%$ (Table 2). The latter results are comparable to the percent differences seen in y-axis measurements with and without foam $(0.0-1.2 \%$ and $0.0-1.7 \%$, respectively). The differences in foam heights did not present discordant error rates. Y-axis measurements showed percent 
differences of $0.56-2.69 \%$ when the bone was placed at an angle and $0.00-0.99 \%$ when the bone was straight. X-axis measurements showed only $0.18 \%$ differences between angled and straight placement.

The Bland-Altman plot shows that most measurement differences between the dry and radiograph bones are within the upper and lower limits of $2 \mathrm{~mm}$ (Fig. 4). Approximately three measurements fall outside the upper and lower limits of agreement, and only one is considered beyond the limits.

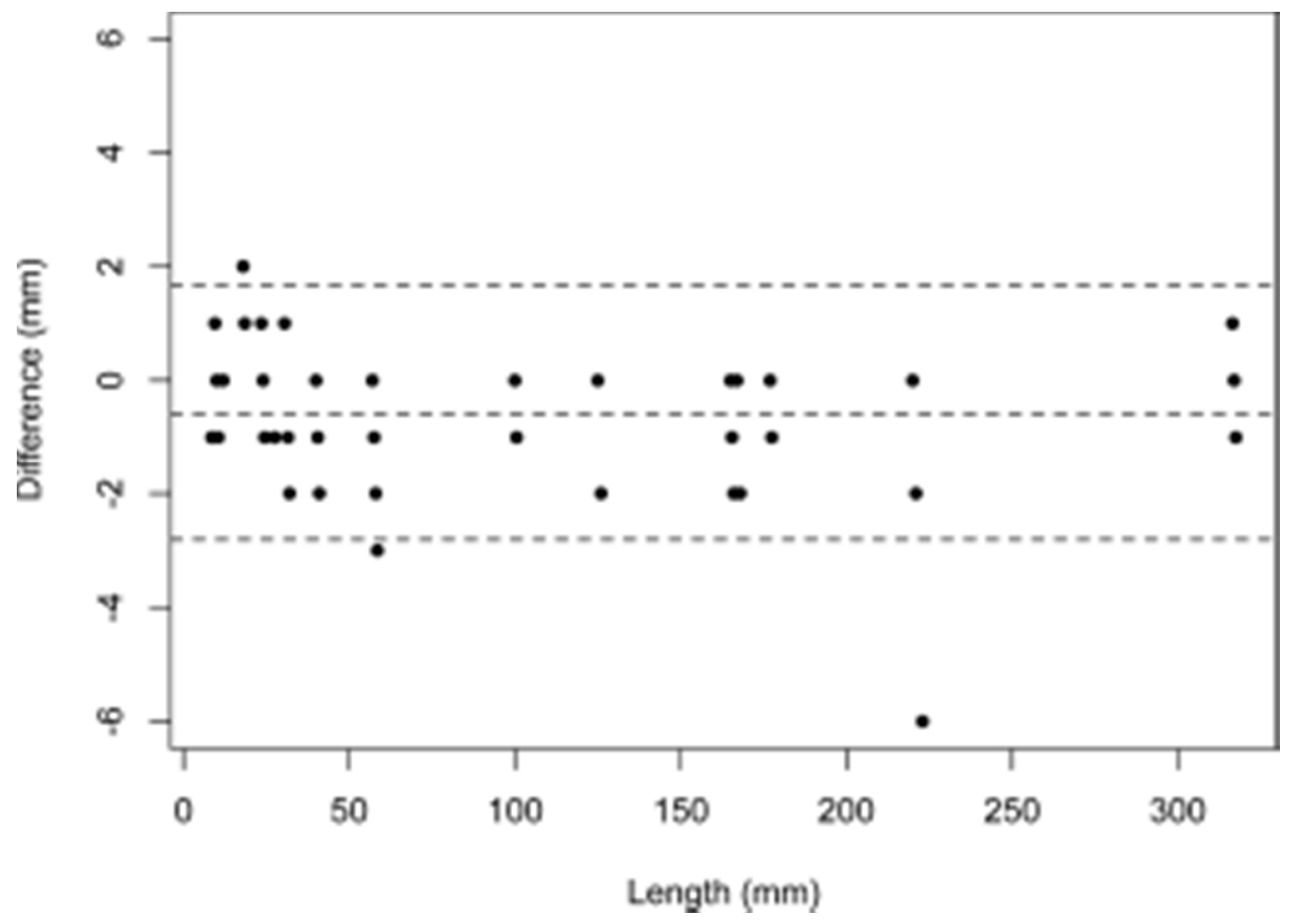

Figure 4 - Bland-Altman plot illustrating the differences between radiographic and dry bone measurements with upper and lower agreement levels.

\section{DISCUSSION}

Adequate levels of measurement error are not clear-cut and tend to relate to the specific variable/object being measured (Ulijaszek and Kerr, 1999; Goto and Mascie-Taylor, 2007). In this study, the three approaches used to measure precision demonstrate that Lodox Statscan images are interchangeable with measurements from dry bone, especially length measurements, or those following the y-axis. Most 
anthropologists accept measurements within $2 \mathrm{~mm}$ when taken on the same object; when following these standards, the percent agreement in this study is very high (97\%).

With all imaging variables combined and as expected for fan-beam geometry, the y-axis measurements have percent differences lower than the x-axis measurements. The percent differences of $y$ axis measurements is analogous to distortion recorded in prospective longitudinal growth studies where SID and OID are controlled for optimal results (Maresh, 1970; Grivas and Komar, 2008). Larger disparities of the $\mathrm{x}$-axis measurements would be expected when imaged in a peripheral table location due to the design of the machine. However, negligible differences were seen between central and peripheral placement in y-axis measurements and between central and peripheral x-axis measurements. The angle was not influential in producing distortion in $\mathrm{x}$-axis measurements as both straight and angled positioning demonstrated similar amounts of error. The increase in error rate of angled y-axis measurements $(0.56-2.69 \%)$ compared with straight $y$-axis measurements $(0.00-0.99 \%)$ may be indicative of $x$-axis distortion since the bone is not parallel to the y-axis. The OID variable introduced the most error $(4.6 \%$ error) for x-axis measurements. Minimal error was found in y-axis measurements with and without foam, and in $\mathrm{x}$-axis measurements without foam.

The Bland-Altman plot illustrates that the majority of comparable measurements fall within the upper and lower agreement levels. The measurement that falls outside the two standard deviations of the BlandAltman plot was obtained from a bone imaged with foam and placed at a $65^{\circ}$ angle, the largest angle of all bones in the study. Although this was a y-axis measurement, the error may due to both angle and OID such that it is more similar to an $\mathrm{x}$-axis measurement. As shown, $\mathrm{x}$-axis measurements and an OID present with the greatest measurement error.

When obtaining measurements from Lodox Statscan images, the x-axis and OID need to be considered. If measurements of large dimensions are taken along the slot (x) axis, the amount of distortion needs to be obtained so as to determine the need for a correction factor. Furthermore, measurements of objects with 
larger soft tissue thicknesses may also present with more distortion, such as the femoral heads or pelvis. The Lodox Statscan software does have a feature for correcting the aspect ratio, which is related to the OID, but this feature has not been validated in its use. The aspect ratio was not applied in this study as it is not a feature used in hospitals and/or forensic laboratories.

Since most studies evaluate measurement error of quantitative variables with correlation coefficients, comparative data for the results of the current study are not readily available. In order to circumvent this problem, percent differences of a craniometric dataset where two observers have measured the same crania and percent differences for intra-observer error of the dry bone sample used in the current study, are applied. While the two datasets present inter- and intra-observer error, respectively, and the radiographic images present distortion error, the goal was to compare percent differences between datasets as a means to assess whether the error in radiographic images is equivalent to the error inherently introduced between observers. The craniometric dataset presents a percent difference range of $0.09-9.80 \%$. Generally, percent differences of the intra-observer dry bone dataset fell between $0.0-4.08 \%$, but there was one midshaft measurement with a $1 \mathrm{~mm}$ difference which resulted in a $10.5 \%$ difference. Although the radiographic measurements had a higher maximum percent difference (inclusive of all imaging variables and $x$ - and $y$-axes) of $11.8 \%$, the distortion error is similar to the error observed in the Lodox Statscan-generated images.

Though the current study was initiated to evaluate the Lodox Statscan-generated images as a means to obtain information on the subadult skeleton, the results are also applicable to adult skeletons. The statistical analyses show that using Lodox Statscan-generated images for metric analyses of long bones are acceptable when the patient is placed in the anatomical position. The observer will need to apply their own judgment as angled y-axis measurements presented with higher error.

The Lodox Statscan machine is utilized in trauma units as part of triage standard operating procedures and forensic laboratories as part of the postmortem examination. There is also one dedicated pediatric installation in Cape Town, South Africa. The daily procedures at both types of institutions result in a large 
data source available to evaluate subadult, as well as adult, skeletal material. Moreover, the opportunity to image a full body with minimal distortion and high resolution could benefit practitioners in many fields as prospective analyses require funding, participants, ethical approval for each candidate, and access to facilities which practitioners may not have. Retrospective data collection is ideal to gather the same type of data without the complexities of a large-scale prospective study. The Lodox Statscan is not invasive, delivers a very low dose and could be a source of image data of modern individuals.

\section{ACKNOWLEDGEMENTS}

Thanks to Belinda Speed for assistance with the UCT Human Skeletal Collection and Prof. Rheeder (University of Pretoria) and Glenn Eanes who both assisted with R code. Three anonymous reviewers offered comments and suggestions which strengthened the manuscript.

\section{REFERENCES}

AlQahtani SJ, Hector MP, and Liversidge HM. 2010. Brief Communication: The London Atlas of Human Tooth Development and Eruption. Am J Phys Anthropol 142:481-490.

Anderson M, Messner M, and Green W. 1964. Distribution Lengths of the Normal Femur and Tibia in Children from One to Eighteen Years of Age. J Bone Jt Surg 46A:1197-1202.

Beningfield S, Potgieter H, Nicol A, Van As S, Bowie G, Hering E, and Latti E. 2003. Report on a new type of trauma full-body digital x-ray machine. Emer Radio 10:23-29.

Bland M, and Altman D. 1986. Statistical Methods for Assessing Agreement Between Two Methods of Clinical Measurement. Lancet:307-310.

Bontrager K. 2001. Textbook of Radiographic Positioning and Related Anatomy. 5th Edition. Philade: Mosby.

Buikstra JE, and Ubelaker DH. 1994. Standards for Data Collection from Human Skeletal Remains: Proceedings of a Seminar at the Field Museum of Natural History. Fayetteville: Arkansas Archaeological Research Series. 
Buschang PH. 1982. Differential long bone growth of children between two months and eleven years. American Journal of Physical Anthropology 58:291-295.

Carleton R, and Adler A. 2001. Principles of Radiographic Imaging. 3rd ed. Stanford, CT: Delmar.

Crowder C, and Austin D. 2005. Age Ranges of Epiphyseal Fusion in the Distal Tibia and Fibula of Contemporary Males and Females. J Forensic Sci 50:1-7.

Evangelopoulos D, Deyle S, Zimmermann H, and Exadaktylos A. 2009. Personal experience with wholebody, low dosage, digital X-ray scanning (LODOX-Statscan) in trauma. Scandinavian Journal of Trauma, Resuscitation and Emergency Medicine 17:41-45.

Fazekas S, and Kósa F. 1978. Forensic Fetal Osteology. Budapest: Akademiai Kiado.

Geeta a, Jamaiyah H, Safiza M, Khor G, Kee C, Ahmad A, Suzana S, Rahmah R, and Faudzi A. 2009. Reliability, technical error of measurements and validity of instruments for nutritional status assessment of adults in Malaysia. Singapore Med J 50:1013-18.

Gindhart PS. 1973. Growth standards for the tibia and radius in children aged one month through eighteen years. American Journal of Physical Anthropology 39:41-48.

Goto R, and Mascie-Taylor N. 2007. Precision of Measurement as a Component of Human Variation. J Physio Anthropol 26:253-256.

Greulich W, and Pyle I. 1959. Radiographic Atlas of Skeletal Development of the Hand and Wrist. 2nd ed. Palo Alto: Stanford University Press.

Grivas C, and Komar D. 2008. Evaluating Methods of Age Estimation of Fetal/Neonate Remains from Radiographs Using a Diverse Autopsy Sample. In: 60th Annual Meeting American Association of Forensic Scientists. February 18-23; Washington, DC.

Hill A. 1939. Fetal Age Assessment by Centers of Ossification. American Journal of Physical Anthropology 24:251-72.

Knobel G, Alexander G, and Bowie G. 2006. Lodox Statscan proves to be invaluable in forensic medicine. South African Medical Journal 96:593-594.

Lewis A, and Garn S. 1960. The Relationship between Tooth Formation and Other Maturational Factors. The Angle Orthodontist 30:70-77.

Maresh M. 1955. Linear growth of long bones of extremities from infancy through adolesence. American Journal of Diseases of Children 89:725-742.

Maresh M. 1970. Measurements from Roentgenograms. In: Human Growth and Development. Springfield, IL: CC. Thomas. p 157-200.

Moore-Jansen PH, Ousley SD, and Jantz RL. 1994. Data Collection Procedures for Forensic Skeletal Material. Knoxville: Department of Anthropology, The University of Tennessee.

Ontell F, Ivanovic M, Ablin D, and Barlow TW. 1996. Bone Age in Children of Diverse Ethnicity. American Journal of Roentgenology 167:1395-1398. 
Ousley S. 1995. Should We Estimate Biological or Forensic Stature? J Forensic Sci 40:768-773.

Scheuer L, and Black S. 2000. Developmental Juvenile Osteology. New York: Elsevier Academic Press.

Smith S, and Buschang P. 2004. Variation in Longitudinal Diaphyseal Long Bone Growth in Children Three to Ten Years of Age. American Journal of Human Biology 16:648-657.

Smith S. 2007. Stature Estimation of 3-10 year-old Children from Long Bone Lengths. J Forensic Sci 52:538-46.

Ulijaszek S, and Kerr D. 1999. Anthropometric Measurement Error and the Assessment of Nutritional Status. Br J Nutr 82:165-177. 